\title{
The expression profile of circANKRD36 and ANKRD36 as diagnostic biomarkers of chronic kidney disease in patients with type 2 diabetes mellitus
}

\author{
Nearmeen M. Rashad ${ }^{1 *}$ D, Mohamed H. Sherif², Amal S. El-Shal ${ }^{3}$ and Mona A. E. Abdelsamad ${ }^{4}$
}

\begin{abstract}
Background: The molecular mechanisms for chronic kidney disease (CKD) remain largely unknown and appear to be multifactorial. In the current study, we aimed to study the circulatory levels of circular ankyrin repeat domain 36 (circANKRD36) and ANKRD36 in Egyptian patients with type 2 diabetes mellitus (T2DM) and CKD and to explore their associations with the progression of CKD. This cross-sectional controlled study enrolled 60 patients with T2DM and 40 controls. Real-time polymerase chain reaction (RT-PCR) and real-time quantitative PCR (RT-qPCR) analyses were used to detect the expression levels of circANKRD36 and ANKRD36.

Results: Our results detected that the relative expression levels of circANKRD36 and ANKRD36 were significantly higher in patients with T2DM compared to controls. CircANKRD36 and ANKRD36 were significantly overexpressed in patients with macroalbuminuria $(0.2316 \pm 0.096,0.0086 \pm 0.0035$, respectively) compared microalbuminuria $(0.1347 \pm$ $0.032,0.0037 \pm 0.0008$, respectively) as well as normoalbuminuria $(0.1261 \pm 0.018,0.0027 \pm 0.0004$, respectively), $p^{<} 0.001^{*}$.

Conclusion: The relative expression levels of circANKRD36 and ANKRD36 were significantly increased in patients with T2DM more specifically in patients with diabetic nephropathy (DN) and macroalbuminuria.
\end{abstract}

Keywords: CKD, UACR, CircANKRD36, Gene expression, eGFR, RT-PCR

\section{Background}

Despite advances in the understanding of diabetes mellitus (DM) risk factors and progression, there has been a steady rise in the global prevalence of DM. Previous numerous studies have demonstrated by the end of 2030, the total number of patients with diabetes will increase and is expected to rise by $55 \%$ [1]. Chronic kidney disease (CKD) is a major health problem. It is considered one of the most predominant chronic complications of type 2 diabetes mellitus (T2DM), and several studies reported that $\sim 30-40 \%$ of patients with DM had CKD [2].

\footnotetext{
* Correspondence: nrashad78@yahoo.com

${ }^{1}$ Internal Medicine Department, Faculty of Medicine, Zagazig University,

Zagazig, Egypt

Full list of author information is available at the end of the article
}

Diabetic nephropathy $(\mathrm{DN})$ is defined as persistently elevated urine albumin creatinine ratio $>30 \mathrm{mg} / \mathrm{g}$ and persistently reduced estimated glomerular filtration rate (eGFR) $<60 \mathrm{ml} / \mathrm{min}$ per $1.73 \mathrm{~m}^{2}[3]$.

Accumulating studies have reported that the progression of CKD seems to be multifactorial, which may be due to interactions between genetic and environmental factors [4]. There are various epigenetic regulators including noncoding RNAs (ncRNAs) such as circular RNAs (circRNAs) [5]. Interesting studies presume that circRNAs regulate hyperglycemia-induced vascular endothelial cell dysfunction [6]. Likewise, another research suggested that hsa_circ_0054633 has a defensive mechanism against hyperglycemia hazards on 
endothelial functions through the miR-218/heme oxygenase-1 axes [7].

An overwhelming amount of evidence has demonstrated that epigenetics are interrelated with various diabetic microvascular complications development and progression, for example, retinopathy, nephropathy, and neuropathy. Consistent with this notion, a recent study explored the pathophysiological roles of circRNAs in patients with T2DM as well as in patients with CKD [8].

There is compelling evidence suggesting that early diagnosis of CKD decreases morbidity and mortality rates. Interestingly, microalbuminuria is the gold standard for the early diagnosis of DN. However, many studies have suggested that it is inadequate and has some drawbacks. At this time, recent technologies and novel biomarkers have been accepted for early diagnosis and progression evaluation of CKD in patients with T2DM [9]. The current study aims to evaluate the expression profile levels of circANKRD36 and ANKRD36 in Egyptian patients with T2DM and CKD and to explore their associations with the progression of CKD.

\section{Methods}

\section{Study population}

The study enrolled 60 patients with T2DM and 40 matched controls of the same ethnic origin. T2DM patients were diagnosed according to the American Diabetes Association criteria, reported in 2020 [10]. We categorized T2DM patients according to the urine albumin creatinine ratio (UACR) into 3 subgroups; normal $(n=38)$ was defined as a UACR persistently $<30 \mathrm{mg} / \mathrm{g}$, microalbuminuria $(n=15)$ as a UACR between 30 and $300 \mathrm{mg} / \mathrm{g}$, and macroalbuminuria $(n=7)$ as a UACR $>300$ $\mathrm{mg} / \mathrm{g}$. All enrolled subjects underwent full history taking and clinical examination with special concern to other comorbidities including hypertension, cerebrovascular stroke, ischemic heart disease, current medications, duration of diabetes, and other diabetic complications. We calculated the body mass index (BMI). The eGFR was calculated by the CKD-EPI equation [11]. The exclusion criteria were for any patients with chronic liver diseases, autoimmune diseases, inflammatory diseases, and infections. We also excluded pregnant females to avoid false results of albuminuria.

\section{Blood samples and biochemical analysis}

Fasting plasma glucose (FPG), total cholesterol (TC), and triglycerides (TG) were measured by routine enzymatic methods (Spinreact). HDLc was determined after precipitation of apo B-containing lipoproteins. LDLc was calculated using the Friedewald formula [12]. The UACR measured in a spot urine sample is highly correlated with the 24-h urine albumin excretion [13].
Total RNA was extracted by using a total RNA Extraction Kit (Cat. No. BSC52S1); supplied by Bioer Technology Co., China. The RNA reverse transcription kit is HiSenScript kit (Cat. No. 25014); supplied by iNtRON Biotechnology, Inc., South Korea, and it was used for cDNA synthesis from the total RNA. The RNA quality was quantified by A260 using UV/spectrophotometer. RT-PCR technique was applied by using StepOne Plus ${ }^{\text {TM }}$ System (Applied Biosystems Inc., USA). RT-PCR was done in $20 \mu \mathrm{l}$ as a final volume containing cDNA $(5 \mu \mathrm{l}), 10 \mu \mathrm{l}$ of $1 \mathrm{X}$ Quantitect sybr green with low ROX PCR master mix (Cat.No.RT500S; Enzynomics, Republic of Korea), and $\mathrm{pmol} / \mathrm{ml}$ each primer $(0.5 \mu \mathrm{l}$ for each) of circANKRD36 or ANKRD36 and was completed by $4 \mu \mathrm{l}$ $\mathrm{DdH}_{2} \mathrm{O}$ according to the manufacturer's instructions.

The primers of circANKRD36 or ANKRD36 were designed by Primer 3 (http://www-genome. ut. ee/), and according to previous research [14], their sequences are listed in Table 1.

Amplification protocol consisted of initial denaturation with polymerase activation at $95^{\circ} \mathrm{C}$ for $15 \mathrm{~min}$, then 40 cycles of denaturation $94^{\circ} \mathrm{C}$ for $15 \mathrm{~s}$; annealing at $55^{\circ} \mathrm{C}$ for $30 \mathrm{~s}$ and extension at $70^{\circ} \mathrm{C}$ for $30 \mathrm{~s}$. Expression levels of each gene were normalized to $\beta$-actin. The relative gene expression of the target ANKRD36 was analyzed and calculated using RT-PCR and the Livaks $2^{-\Delta \Delta \mathrm{Ct}}$ equation relation to $\beta$-actin as internal control genes. The $\mathrm{Ct}$ (threshold cycle) is a relative measure of the concentration of the target in the PCR reaction. $\triangle \Delta C T=$ [CT(target, normal)-CT(I.C, normal)]-[CT(target, HCC)-CT(I.C, HCC)].

\section{Statistical analysis}

Analysis of data was performed using SPSS v.21. The data were expressed using descriptive statistics (mean \pm standard deviation) and were analyzed using the $t$ test. Comparisons between the two groups were performed using chi-square $\left(X^{2}\right)$. We tested the association between the expression profile of circANKRD36 and ANKRD36 with UACR and other studied variables in patients with CKD and detected the independent variables by linear regression. Receiver operating characteristics (ROC) tested the diagnostic powers of circANKRD36 and its target gene ANKRD36 levels among studied subjects.

\section{Results}

Study was conducted on 60 Egyptian patients with T2DM-42 were females (70\%), 18 were males (30\%), and their mean age was $43.63 \pm 12.17$ years-in addition to 40 healthy subjects as control-25 were females $(62.5 \%), 15$ were males (37.5\%), and their mean age was $41.55 \pm 10.45$ years. The diabetic and control groups were matched for age, sex, and smoking. According to the current study results, patients with T2DM had 
Table 1 Primers of circANKRD36 or ANKRD36

\begin{tabular}{lll}
\hline Gene & Forward primer(5'-3') & Reverse primer (5'-3') \\
\hline circANKRD36 & GGAGGCCACAAGTGATGAGA & CCTGGTGGTTTCTCAGAAGAC \\
ANKRD36 & CGAGGAAGATTCTGTTTTGGGT & CCTTCTCATCACTTGTGGCC \\
$\beta$-actin & TTCCTTCCTGGGCATGGA & GAGGAGCAATGATCTTGA \\
\hline
\end{tabular}

higher values of metabolic risk factors and impairment of kidney function compared to the control group as shown in Table 2, $p^{<} 0.001^{*}$.

\section{General characteristics of diabetic patients}

The results revealed that 38 patients had normal UACR, 15 patients had microalbuminuria, and 7 patients had macroalbuminuria. Thus, about $36.7 \%$ of patients with T2DM had DN. There was a statistically significant long duration of diabetes as well as higher values of BMI, waist/hip ratio, diastolic blood pressure, TG, and UACR in patients with macroalbuminuria compared to other subgroups. Regarding diabetic treatment and diabetic vascular complications, there was a statistically significant difference between the studied groups, $p{ }^{<} 0.001^{*}$, Table 3.

\section{Relative expression of circANKRD36 and mRNA} expression level of ANKRD36 in the studied groups

The results of the current study show significantly higher values of circANKRD36 relative expression level in the T2DM group compared to the control group $\left(0.219 \pm 0.061\right.$, vs $\left.0.111 \pm 0.013 ; p^{<} 0.001\right)$. Regarding the mRNA expression level of ANKRD36, it was higher in the T2DM than that in the control group $(0.006454 \pm$ 0.0018 , vs. $0.0033 \pm 0.004, p^{<} 0.001$ ), Table 2 .

\section{Relative expression of circANKRD36 and mRNA expression level of ANKRD36 in patients with T2DM}

Our results show that patients with macroalbuminuria $(0.2316 \pm 0.096)$ had statistically significantly higher values of the relative expression level of circANKRD36 compared to patients with microalbuminuria $(0.1347 \pm$ $0.032)$ and patients with normoalbuminuria $(0.1261 \pm$ $0.018)$, Fig. 1a, $p^{<} 0.01$. Regarding the mRNA expression level of $A N K R D 36$, patients with macroalbuminuria $(0.0086 \pm 0.0035)$ had statistically significantly higher values of the relative expression level of circANKRD36 compared to patients with microalbuminuria $(0.0037 \pm$ $0.0008)$ and patients with normoalbuminuria $(0.0027 \pm$ $0.0004)$, Fig. $1 \mathrm{~b}, p^{<} 0.01$.

Correlations between relative expression of circANKRD36 and the mRNA expression level of ANKRD36 with clinical and laboratory characteristics in patients with CKD

The current study revealed that the relative expression of circANKRD36 and the mRNA expression level of ANKRD36 were significantly positively correlated with

Table 2 Anthropometric and biochemical characteristics of the studied groups

\begin{tabular}{|c|c|c|c|}
\hline Variables & Control group $(n=40)$ & T2DM patients $(n=60)$ & $P$ value \\
\hline Body mass index $\left(\mathrm{kg} / \mathrm{m}^{2}\right)$ & $22.18 \pm 1.289$ & $36.03 \pm 4.26$ & ${ }^{<} 0.001^{*}$ \\
\hline Waist/hip ratio & $0.864 \pm 0.121$ & $1.17 \pm 0.31$ & ${ }^{<} 0.001^{*}$ \\
\hline Systolic blood pressure & $117.5 \pm 8.40$ & $149.7 \pm 20.54$ & $<0.001^{*}$ \\
\hline Diastolic blood pressure & $75.6 \pm 4.589$ & $92.92 \pm 14.33$ & ${ }^{<} 0.001^{*}$ \\
\hline Total cholesterol (mg/dl) & $184.3 \pm 19.90$ & $222.88 \pm 28.1$ & $<0.001^{*}$ \\
\hline Triglycerides (mg/dl) & $175.26 \pm 31.019$ & $276.16 \pm 58.6$ & $<0.001^{*}$ \\
\hline LDL cholesterol (mg/dl) & $100.08 \pm 23.067$ & $124.91 \pm 32.4$ & $<0.001^{*}$ \\
\hline HDL cholesterol (mg/dl) & $56.48 \pm 4.87$ & $35.25 \pm 5.63$ & $<0.001^{*}$ \\
\hline $\mathrm{FPG}(\mathrm{mg} / \mathrm{dl})$ & $89.72 \pm 6.304$ & $220.97 \pm 30.04$ & $<0.001^{*}$ \\
\hline $\mathrm{HbA1c}(\%)$ & $5.63 \pm 0.624$ & $9.59 \pm 2.206$ & $<0.001^{*}$ \\
\hline eGFR (mL/min) & $93.37 \pm 7.56$ & $76.19 \pm 13.2$ & $<0.001^{*}$ \\
\hline Serum creatinine (mg/dl) & $0.916 \pm 0.27$ & $1.46 \pm 0.48$ & $<0.001^{*}$ \\
\hline UACR $(\mathrm{mg} / \mathrm{g})$ & $20.72 \pm 2.304$ & $122.97 \pm 103.04$ & ${ }^{<} 0.001^{*}$ \\
\hline Relative expression of circANKRD36 & $0.111 \pm 0.013$ & $0.219 \pm 0.061$ & $<0.001^{*}$ \\
\hline mRNA expression level of ANKRD36 & $0.0033 \pm 0.004$ & $0.006454 \pm 0.0018$ & $<0.001^{*}$ \\
\hline
\end{tabular}

T2DM Type 2 diabetes mellitus, FPG Fasting plasma glucose, HbA1c Hemoglobin, A1c Estimated glomerular filtration rate, UACR Urine albumin: creatinine ratio, circANKRD36 Circular ankyrin repeat domain 36 ${ }^{*} P<0.05$ 
Table 3 Laboratory and anthropometric parameters of diabetic patients

\begin{tabular}{|c|c|c|c|c|c|c|}
\hline Variables & Normal $(n=38)$ & Microalbuminuria $(n=15)$ & Macroalbuminuria $(n=7)$ & P1 & P2 & P3 \\
\hline Duration of diabetes (years) & $8.864 \pm 3.1$ & $10.87 \pm 3.31$ & $15.17 \pm 6$ & ${ }^{<} 0.001^{*}$ & ${ }^{<} 0.001^{*}$ & $<0.001^{*}$ \\
\hline Body mass index $\left(\mathrm{kg} / \mathrm{m}^{2}\right)$ & $35.18 \pm 1.289$ & $37.4 \pm 4.3$ & $38.2 \pm 4.26$ & $<0.001^{*}$ & ${ }^{<} 0.001^{*}$ & 0.891 \\
\hline Waist/hip ratio & $0.964 \pm 0.121$ & $1.27 \pm 0.31$ & $1.27 \pm 0.31$ & ${ }^{<} 0.001^{*}$ & ${ }^{\circ} 0.001^{*}$ & $<0.001^{*}$ \\
\hline Systolic blood pressure & $140.5 \pm 8.43$ & $145.7 \pm 12.2$ & $140.7 \pm 14.3$ & 0.878 & 0.943 & 0.970 \\
\hline Diastolic blood pressure & $85.6 \pm 4.58$ & $99.9 \pm 14.3$ & $105.9 \pm 14.3$ & $<0.001^{*}$ & $<0.001^{*}$ & 0.839 \\
\hline Total cholesterol (mg/dl) & $216.3 \pm 19.9$ & $232.8 \pm 25.1$ & $234.8 \pm 48.1$ & $<0.001^{*}$ & 0.128 & 0.914 \\
\hline Triglycerides (mg/dl) & $175.3 \pm 31.1$ & $266.4 \pm 37.6$ & $285.3 \pm 44.6$ & $<0.001^{*}$ & $<0.001^{*}$ & 0.800 \\
\hline LDL cholesterol (mg/dl) & $111.1 \pm 26.2$ & $114.5 \pm 36.4$ & $130.9 \pm 37.4$ & 0.093 & 0.135 & 0.824 \\
\hline HDL cholesterol (mg/dl) & $36.9 \pm 4.87$ & $32.25 \pm 1.5$ & $31.25 \pm 3.4$ & $<0.001^{*}$ & 0.055 & 0.694 \\
\hline $\mathrm{FPG}(\mathrm{mg} / \mathrm{dl})$ & $227.1 \pm 16.3$ & $231.9 \pm 31.4$ & $265.9 \pm 41.3$ & 0.077 & 0.886 & 0.289 \\
\hline HbAlc (\%) & $8.63 \pm 1.4$ & $9.59 \pm 2.3$ & $10.59 \pm 1.5$ & $<0.001^{*}$ & 0.222 & 0.447 \\
\hline eGFR (mL/min) & $93.37 \pm 7.5$ & $76.19 \pm 13.2$ & $76.19 \pm 13.2$ & $<0.001^{*}$ & 0.135 & 0.772 \\
\hline Serum creatinine $(\mathrm{mg} / \mathrm{dl})$ & $1.216 \pm 0.27$ & $1.46 \pm 0.9$ & $2.36 \pm 1.2$ & 0.250 & ${ }^{<} 0.001^{*}$ & $<0.001^{*}$ \\
\hline UACR (mg/g) & $25.7 \pm 3.3$ & $183.97 \pm 58.1$ & $358.7 \pm 94.3$ & ${ }^{<} 0.001^{*}$ & $<0.001^{*}$ & $<0.001^{*}$ \\
\hline \multicolumn{7}{|l|}{ Medication } \\
\hline Diet & $8(21.1 \%)$ & $7(46.7 \%)$ & $6(85.7 \%)$ & ${ }^{<} 0.001^{*}$ & ${ }^{\circ} 0.001^{*}$ & $<0.001^{*}$ \\
\hline Oral & $8(21.1 \%)$ & $7(46.7 \%)$ & $5(71.4 \%)$ & $<0.001^{*}$ & ${ }^{\circ} 0.001^{*}$ & $<0.001^{*}$ \\
\hline Insulin & $19(50 \%)$ & $9(60 \%)$ & $5(71.4 \%)$ & $<0.001^{*}$ & $<0.001^{*}$ & $<0.001^{*}$ \\
\hline \multicolumn{7}{|l|}{ Vascular complications } \\
\hline Retinopathy & $14(36.8 \%)$ & $8(53.3 \%)$ & $7(100 \%)$ & ${ }^{<} 0.001^{*}$ & ${ }^{<} 0.001^{*}$ & $<0.001^{*}$ \\
\hline Neuropathy & $20(52.6 \%)$ & $10(66.7 \%)$ & $5(71.4 \%)$ & $<0.001^{*}$ & $<0.001^{*}$ & $<0.001^{*}$ \\
\hline Stroke & $6(15.8 \%)$ & $8(53.3 \%)$ & $6(85.7 \%)$ & $<0.001^{*}$ & ${ }^{<} 0.001^{*}$ & $<0.001^{*}$ \\
\hline $\mathrm{CHD}$ & $14(36.8 \%)$ & $8(53.3 \%)$ & $7(100 \%)$ & $<0.001^{*}$ & $<0.001^{*}$ & $<0.001^{*}$ \\
\hline
\end{tabular}

T2DM Type 2 diabetes mellitus, FPG Fasting plasma glucose, HbA1c Hemoglobin, A1C Estimated glomerular filtration rate, UACR Urinary albumin-creatinine ratio, circANKRD36 Circular ankyrin repeat domain 36 ${ }^{*} P<0.05$

duration of diabetes, diastolic blood pressure, TG, LDL, FPG, HbA1c, creatinine, and UACR and were significantly negatively correlated with eGFR, $p<0.001$, Table 4 .

\section{Linear regression analysis in patients with CKD}

A linear regression analysis test was done to assess the main independent parameters associated with the relative expression of circANKRD36 and the mRNA expression level of ANKRD36. Our results showed that eGFR as well as UACR were independently correlated with both epigenetic biomarkers, $p<0.001$, Table 5 .

\section{Accuracy of relative expression of circANKRD36 and mRNA expression level of ANKRD36 for discriminating patients with T2DM from the control group by ROC analysis}

We investigated the potential diagnostic value of the relative expression of circANKRD36 (Fig. 2a) and the mRNA expression level of ANKRD36 (Fig. 2b) by the ROC test. When we discriminate patients with T2DM from the control group, the cutoff values were 0.142 and 0.0048 , respectively, and the AUC was 0.932
(95\% CI $=0.875-0.989)$ and 0.945 (95\% CI $=0.887-$ 0.992); additionally, the sensitivities and the specificities were $90 \%$ and $93 \%$ for circANKRD36 and $88.3 \%$ and $87.5 \%$ for the mRNA expression level of $A N K R$ D36, respectively.

\section{Accuracy of relative expression of circANKRD36 and the mRNA expression level of ANKRD36 for discriminating patients with microalbuminuria from patients with normoalbuminuria by ROC analysis}

We further investigated the potential diagnostic value of the relative expression of circANKRD36 (Fig. 3a) and the mRNA expression level of ANKRD36 (Fig. 3b) by the ROC test. When we discriminate patients with microalbuminuria from patients with normal UACR group, the cutoff values were 0.1338 and 0.0054 , respectively. The AUC was 0.762 (95\% CI $=0.615-0.909)$ and 0.895 (95\% $\mathrm{CI}=0.7609-1.000)$. Additionally, the sensitivities and the specificities were $81.8 \%$ and $69.4 \%$ for circANKRD36 and $85.7 \%$ and $87.7 \%$ for the mRNA expression level of $A N K R D 36$, respectively, $p<0.001$. 

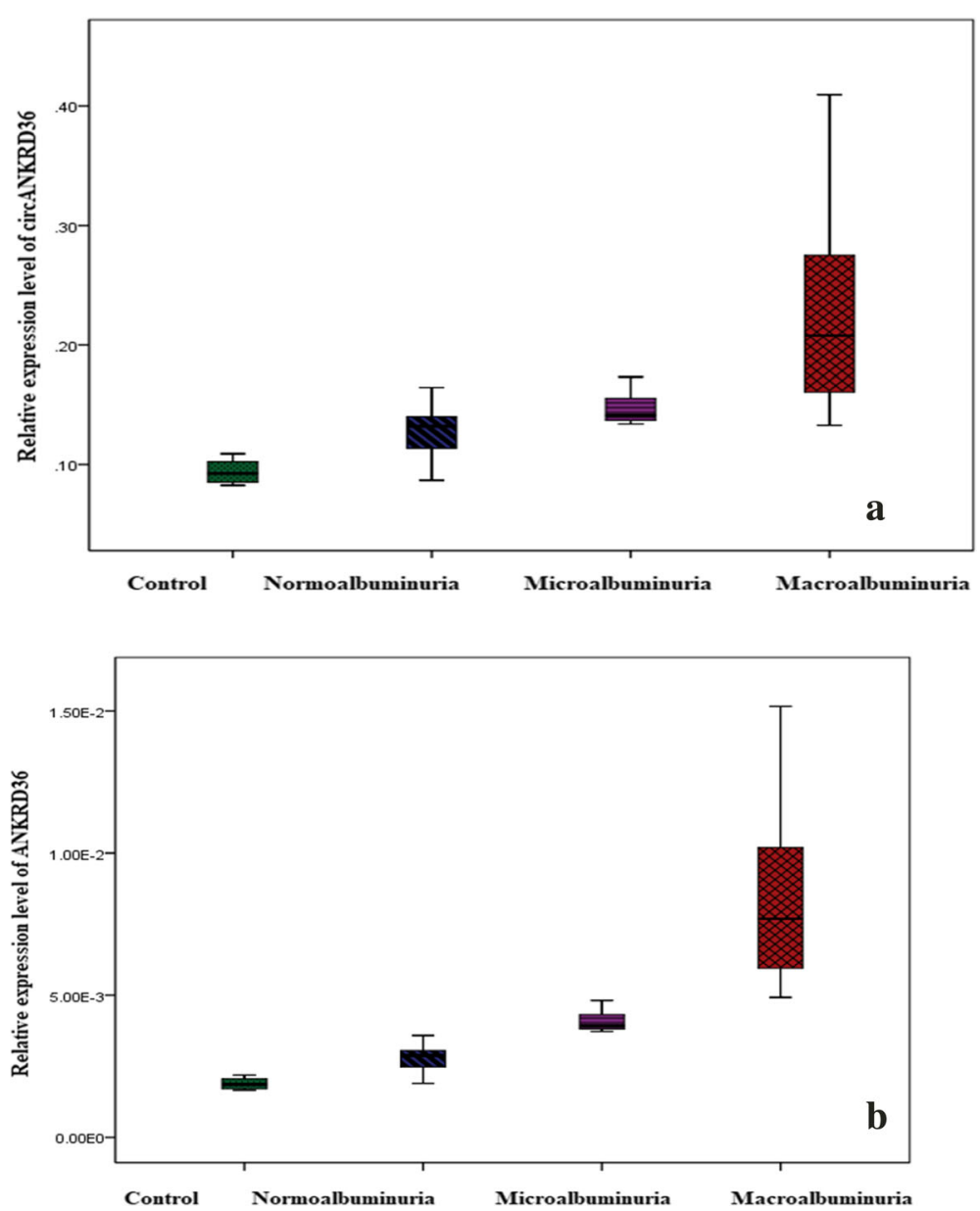

Fig. 1 a The relative expression levels of circANKRD36 in the studied groups. b Relative expression levels of ANKRD36 in the studied groups

\section{Accuracy of relative expression of circANKRD36 and mRNA expression level of ANKRD36 for discriminating patients with macroalbuminuria from patients with microalbuminuria by ROC analysis}

The relative expression of circANKRD36 (Fig. 4a) and the mRNA expression level of ANKRD36 (Fig. 4b) were done by the ROC test. When we discriminate patients with microalbuminuria from patients with normal UACR group, the cutoff values were 0.1782 and 0.0064 , respectively. The AUC was 0.819 (95\% CI $=0.561-1.000)$ and $0.781(95 \% \mathrm{CI}=0.611-0.909)$. In addition, the sensitivities and the specificities were $80.1 \%$ and $80.7 \%$ for circANKRD36 and $85.7 \%$ and $87.7 \%$ for the mRNA expression level of $A N K R D 36$, respectively, $p<0.001$.

\section{Discussion}

Diabetes is a common chronic metabolic disease. Mounting evidence has elaborated that the major causes of morbidity and mortality in the diabetic population are retinopathy, nephropathy, cardiomyopathy, neuropathy, and atherosclerosis. These observations suggested that the macro- and microvascular affections are key features of such complications [15]. Growing evidence has illustrated that low-grade inflammatory changes associated with increased production of inflammatory mediators may be a key mechanism in the pathogenesis of chronic diabetic complications [16].

In the present study, we observed that out of sixty Egyptian patients with T2DM, twenty-two patients had DN with microalbuminuria $(n=15)$ and seven patients had macroalbuminuria. Thus, about $36.7 \%$ of patients with T2 diabetes mellitus had DN.

Similar to our result, a study conducted by Zelmanovitz et al. observed that about $30 \%$ of patients with T2DM develop clinically overt nephropathy [17]. DN is a multifactorial disorder that results from the interaction between environmental and genetic factors. Hyperglycemia, hypertension, and proteinuria are the main insults that cause structural abnormalities in a DN [18].

According to Elnajjar et al. study, about $78 \%$ of all the patients studied had DN. This study was conducted on both patients with T1DM and T2DM. Thus, the high 
Table 4 Correlations between relative expression of circANKRD36 and the mRNA expression level of ANKRD36 with clinical and laboratory characteristics in patients with CKD

\begin{tabular}{|c|c|c|c|c|}
\hline \multirow[t]{2}{*}{ Variables } & \multicolumn{2}{|c|}{ circANKRD36 } & \multicolumn{2}{|c|}{ ANKRD36 } \\
\hline & $r$ & $p$ & $r$ & $p$ \\
\hline Duration of diabetes (years) & 0.460 & $<0.001^{*}$ & 0.511 & $<0.001^{*}$ \\
\hline Body mass index $\left(\mathrm{kg} / \mathrm{m}^{2}\right)$ & 0.246 & 0.058 & 0.044 & 0.741 \\
\hline Waist/hip ratio & 0.139 & 0.290 & 0.102 & 0.440 \\
\hline Systolic blood pressure & 0.207 & 0.112 & 0.056 & 0.666 \\
\hline Diastolic blood pressure & 0.282 & $<0.001^{*}$ & 0.380 & $<0.001^{*}$ \\
\hline Total cholesterol (mg/dl) & 0.117 & 0.372 & 0.180 & 0.058 \\
\hline Triglycerides (mg/dl) & 0.383 & $<0.001^{*}$ & 0.346 & $<0.001^{*}$ \\
\hline LDL cholesterol (mg/dl) & 0.371 & $<0.001^{*}$ & 0.453 & $<0.001^{*}$ \\
\hline HDL cholesterol (mg/dl) & -0.039 & 0.770 & -0.081 & 0.537 \\
\hline FPG (mg/dl) & 0.459 & $<0.001^{*}$ & 0.522 & $<0.001^{*}$ \\
\hline $\mathrm{HbA1c}(\%)$ & 0.345 & $<0.001^{*}$ & 0.508 & $<0.001^{*}$ \\
\hline eGFR (mL/min) & -0.484 & $<0.001^{*}$ & -0.654 & $<0.001^{*}$ \\
\hline Serum creatinine (mg/dl) & 0.567 & $<0.001^{*}$ & 0.493 & $<0.001^{*}$ \\
\hline UACR (mg/g) & 0.589 & $<0.001^{*}$ & 0.840 & $<0.001^{*}$ \\
\hline
\end{tabular}

prevalence of DN among their studied groups could be due to the inclusion of patients with T1DM [19]. However, in our study, we enrolled results in patients with T2DM only. In most type 2 diabetics, the prevalence of DN ranges from 30 to $50 \%$ [20].

Our study revealed clear evidence that in patients with macroalbuminuria, there was a statistically significant long duration of diabetes as well as higher values of
BMI, waist/hip ratio, diastolic blood pressure, TG, and UACR compared to patients with normoalbuminuria and microalbuminuria.

Similar to our results, a study conducted by Elnajjar et al. revealed that there were statistically significant long duration of diabetes, higher systemic blood pressures, an evident decrease in eGFR, poor glycemic control, and obesity in patients with DN compared to patients without DN [21]. In agreement with our results, Kathryn et al. observed that there were significant correlations between the increasing duration of diabetes and the development of microproteinuria [22].

Regarding glycemic control, it was notable that in our observation patients with microalbuminuria had higher HbA1c values compared to patients with normoalbuminuria levels. Similar results were described in the ADVA NCE Collaborative Group [22]. Nevertheless, the Veteran's Affairs Diabetes Trial (VADT) observed that intensive therapy did not lead to a reduction in retinopathy or major nephropathy outcomes compared with standard therapy, but this study was for a short duration [23]. Mounting evidence has elaborated that $\mathrm{BMI}$ values were higher in patients with $\mathrm{DN}[18,24,25]$.

Despite the limited knowledge on the molecular mechanisms underlying the pathophysiology of microvascular complications of T2DM in particular CKD, emerging scientific evidence has indicated that the dysregulated epigenetic is associated with risk and progression of diabetic microvascular complications and could be used as predictors and diagnostic genetic markers of T2DM and CKD.

Table 5 linear regression analyses to test the influence of the main independent variables against relative expression of circANKRD36 and the mRNA expression level of ANKRD36 levels (dependent variable) in patients with CKD

\begin{tabular}{|c|c|c|c|c|c|c|c|}
\hline \multirow[t]{2}{*}{ Model } & \multicolumn{2}{|c|}{ Unstandardized coefficients } & \multirow{2}{*}{$\begin{array}{l}\text { Standardized coefficients } \\
\text { Beta }\end{array}$} & \multirow[t]{2}{*}{$t$} & \multirow[t]{2}{*}{$P$} & \multicolumn{2}{|l|}{$95 \%$ C.I. } \\
\hline & $\bar{B}$ & SE & & & & Lower bound & Upper bound \\
\hline \multicolumn{8}{|l|}{ CircANKRD36 } \\
\hline (Constant) & 0.009 & 0.058 & & 0.151 & 0.881 & -0.108 & 00.126 \\
\hline eGFR & 0.042 & .013 & 0.406 & 3.288 & 0.002 & 0.016 & 0.068 \\
\hline S. creatinine & 0.000 & 0.000 & 0.054 & 0.514 & 0.610 & -0.001 & 0.001 \\
\hline UACR & 0.000 & 0.000 & 0.440 & 3.336 & 0.002 & 0.000 & 0.000 \\
\hline TG & 0.001 & 0.001 & 0.211 & 1.034 & 0.306 & -0.001 & 0.002 \\
\hline \multicolumn{8}{|l|}{ ANKRD36 } \\
\hline (Constant) & -0.001 & 0.002 & & -0.636 & 0.528 & 0.006 & 0.003 \\
\hline eGFR & 0.002 & 0.000 & 0.385 & 3.817 & 0.000 & 0.001 & 0.003 \\
\hline S. creatinine & $-1.100 \mathrm{E}-6$ & 0.000 & -0.006 & -00.075 & 0.941 & 0.000 & 0.000 \\
\hline UACR & $1.012 \mathrm{E}-5$ & 0.000 & 0.555 & 5.144 & 0.000 & 0.000 & 0.000 \\
\hline $\mathrm{HbA1c}$ & $2.228 \mathrm{E}-5$ & 0.000 & 0.151 & 0.907 & 0.369 & 0.000 & 0.000 \\
\hline $\mathrm{TG}$ & 0.002 & 0.002 & 0.245 & 1.025 & 0.310 & -0.002 & 0.007 \\
\hline
\end{tabular}



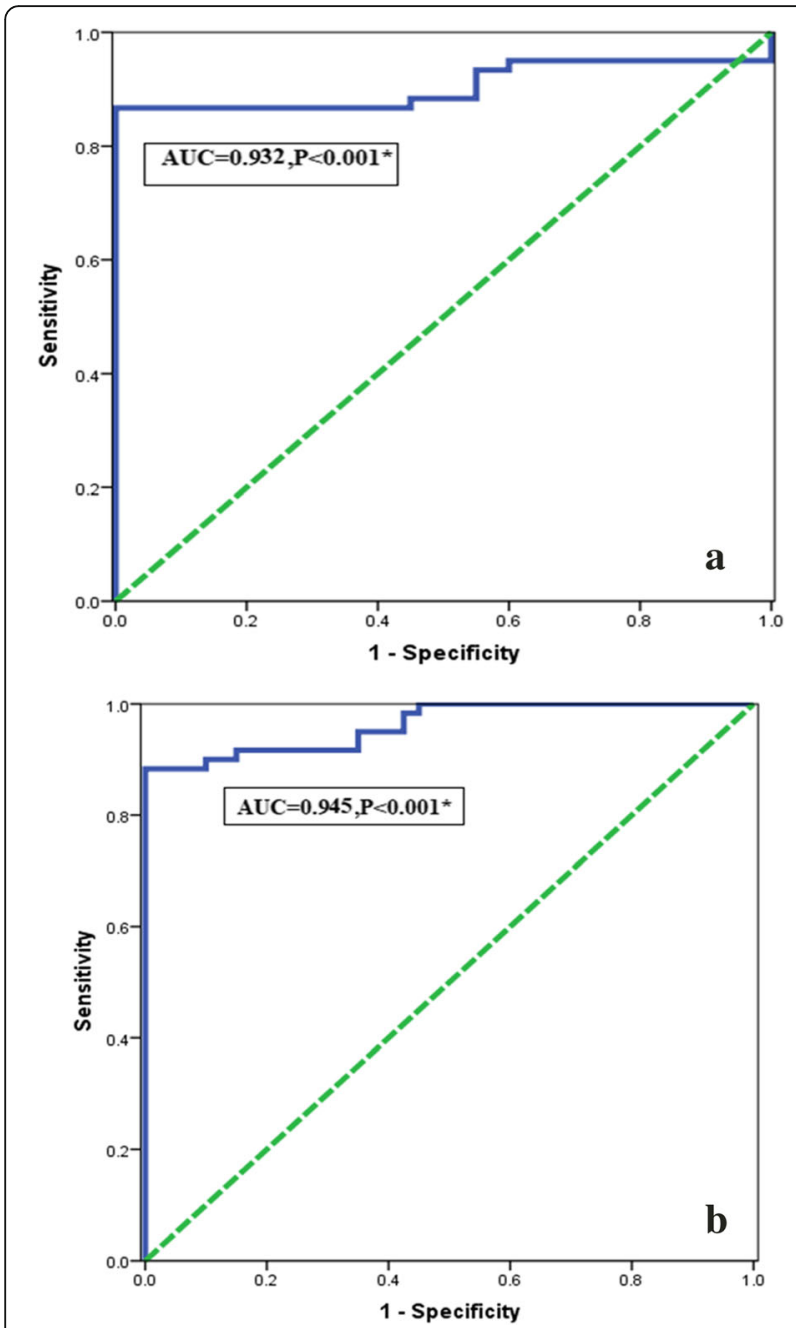

Fig. 2 a The accuracy of the relative expression levels of circANKRD36 for discriminating patients with T2DM from the control group by ROC analysis. $\mathbf{b}$ The accuracy of the relative expression levels of ANKRD36 for discriminating patients with T2DM from the control group by ROC analysis

Emerging evidence demonstrated that about 30\% of patients with T2DM develop clinically overt nephropathy. Consequently, early detection of DN is very useful for preventing progression to renal failure. Due to the high stability of ncRNAs in body fluids, they could be used as a noninvasive diagnostic biomarker for DN. Thus, the current study aimed to investigate the expression profile levels of circANKRD36 and ANKRD36 in Egyptian patients with T2DM and DN and to explore their associations with the risk and the progression of DN.

The results presented herein are innovative as this study performs a robust estimation of the expression levels of circANKRD36 and ANKRD36 in patients with T2DM in particular patients with DN. Expression of circANKRD36 and ANKRD36 were significantly higher in patients with T2DM


Fig. 3 a The accuracy of the relative expression levels of circANKRD36 for discriminating patients with microalbuminuria from patients with normoalbuminuria by ROC analysis. b The accuracy of the relative expression levels of ANKRD36 for discriminating patients with microalbuminuria from patients with normoalbuminuria by ROC analysis

compared to healthy control. Intriguingly, patients with macroalbuminuria had statistically significantly higher values of the relative expression level of circANKRD36 and ANKRD36 compared to patients with microalbuminuria and patients with normoalbuminuria levels.

In agreement with the present study, Fang et al. confirmed that the expression level of circANKRD36 is upregulated in patients with T2DM. Moreover, the level of ANKRD36 which is the host gene of circANKRD36 was upregulated in the T2DM group compared to the general population [26]. Previously, it was shown that ANKR D36 is the host gene of circANKRD36 and the level was increased in the T2DM group as the circ-RNAs can positively regulate their parent genes at the transcriptional level and post-transcriptional level [27-29]. 

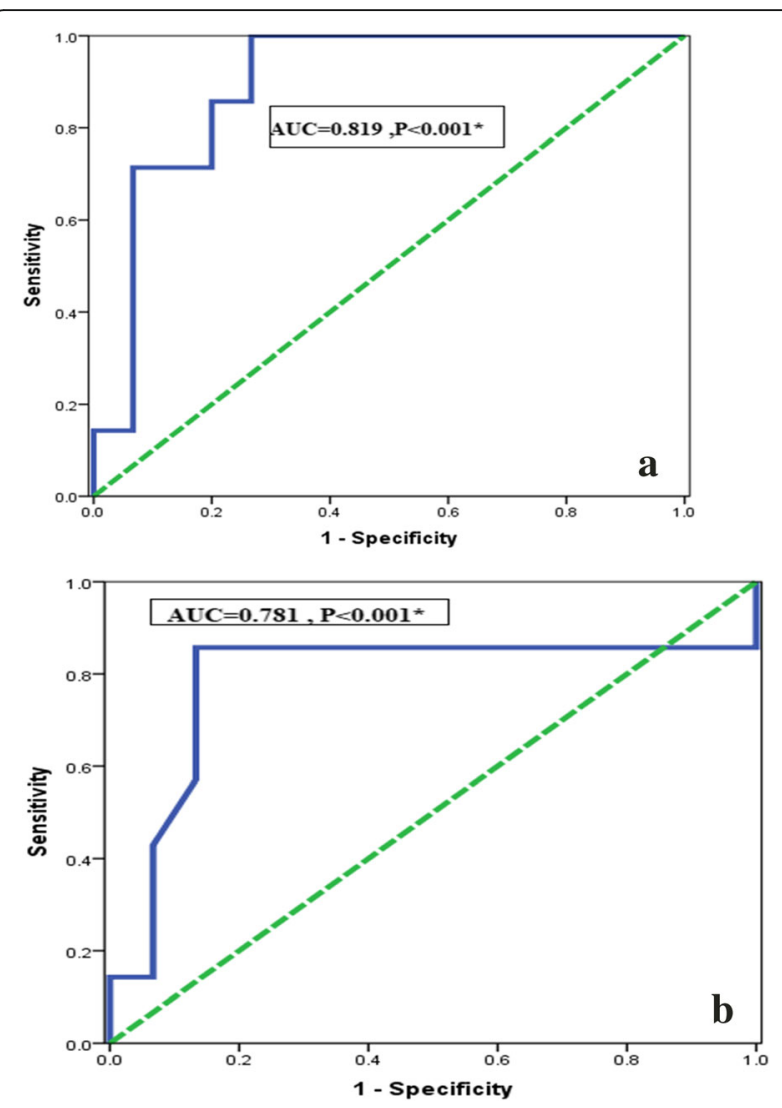

Fig. 4 a The accuracy of the relative expression levels of circ. ANKRD36 for discriminating patients with macroalbuminuria from patients with microalbuminuria by ROC analysis. $\mathbf{b}$ The accuracy of the relative expression levels of ANKRD36 for discriminating patients with macroalbuminuria from patients with microalbuminuria by ROC analysis

The current study revealed that the relative expression of circANKRD36 and mRNA expression levels of ANKRD36 were positively correlated with duration of diabetes, diastolic blood pressure, TG, LDL, FPG, HbA1c, creatinine, and UACR and were significantly negatively correlated with eGFR. Currently, several pieces of evidence exist, demonstrating that miRNAs are involved in the regulation of vascular inflammation [30]. Furthermore, it has been also proposed that circRNAs influence the cardiometabolic risk factors which have a deleterious effect on blood vessels and can lead to the development of endothelial dysfunction and hence diabetic vascular complications [31].

Until the time of writing this article, only one experimental study was conducted to illuminate the roles of circRNA in CKD progression. In this study, circRNA_ 15698 upregulates TGFB1 expression and promotes ECM protein production. Thus, the inhibition of circRNA_15698 could delay the progression of CKD [32].

The current study aims to assess the correlations between circANKRD36 and ANKRD36 relative expression in patients with $\mathrm{DN}$. We observed that, there were significant positive correlations between the levels of both genes and duration of diabetes, diastolic blood pressure, TG, LDL, FPG, HbA1c, creatinine, and UACR. However, there were significant negative correlations between both epigenetics and eGFR. A linear regression test was done to investigate the main independent parameters associated with the relative expression of circANKRD36 and the mRNA expression level of $A N K R$ $D 36$, and we observed that eGFR as well as UACR were independently correlated with both epigenetic biomarkers. Similar results were observed in a significant positive correlation between circANKRD36 and blood glucose as well as Hba1c [26].

ROC analysis was used to clarify the diagnostic power of circANKRD36 and the mRNA expression level of ANKR $D 36$ for discriminating patients with T2DM from the control group. Our results revealed that the sensitivities and the specificities were $90 \%$ and $93 \%$ for circANKRD36 besides $88.3 \%$ and $87.5 \%$ for the mRNA expression level of ANKRD36. As regards, the differentiation of patients with microalbuminuria from patients with normoalbuminuria, the sensitivities and the specificities were $81.8 \%$ and $69.4 \%$ for circANKRD36 as well as $85.7 \%$ and $87.7 \%$ for the mRNA expression level of ANKRD36, respectively. We attempted to discriminate patients with macroalbuminuria from patients with microalbuminuria, the sensitivities, and the specificities were $80.1 \%$ and $80.7 \%$ for circANKRD36 in addition $85.7 \%$ and $87.7 \%$ for the mRNA expression level of ANKRD36, respectively.

\section{Conclusion}

The current study revealed that about $36.7 \%$ of patients with T2DM had DN. The relative expression levels of circANKRD36 and ANKRD36 were significantly higher in patients with T2DM and DN more specifically in patients with macroalbuminuria.

\section{Abbreviations}

BMl: Body mass index; CircANKRD36: Circ. ankyrin repeat domain36; CircRNAs: Circular RNAs; CKD: Chronic kidney disease; DM: Diabetes mellitus; DN: Diabetic nephropathy; eGFR: Estimated glomerular filtration rate; FPG: Fasting plasma glucose; NcRNAs: Noncoding RNAs; RT-qPCR: Real-time quantitative PCR; TC: Total cholesterol; TG: Triglycerides; T2DM: Type 2 diabetes mellitus; UACR: Urine albumin: creatinine ratio

\section{Acknowledgements}

Not applicable.

\section{Authors' contributions}

NMR and MHS collected patients' samples and clinical data. ASE and MAEA prepared the sample for laboratory investigations. NMR wrote the paper. Statistical analysis, interpretation of data, and preparation of the paper for international submission were done by NMR. Critical revision of the manuscript was performed by all of the authors. The authors have read and approved the manuscript. 


\section{Availability of data and materials}

The data that support the findings of this study are available from the corresponding author (nrashad78@yahoo.com) upon reasonable request.

\section{Declarations}

\section{Ethics approval and consent to participate}

The enrolled subjects accepted their participation in the study and gave their written consent. The study protocol was approved by the Ethics Committee of Faculty of Medicine, Zagazig University, and the reference number was IRB (Ethics number. 5144).

\section{Consent for publication}

The authors declare that they received consents for publication from all the participants.

\section{Competing interests}

The authors declare that they have no competing interests.

\section{Author details}

'Internal Medicine Department, Faculty of Medicine, Zagazig University, Zagazig, Egypt. ${ }^{2}$ Organic Chemistry, Faculty of Science, Zagazig University, Zagazig, Egypt. ${ }^{3}$ Medical Biochemistry Department, Faculty of Medicine, Zagazig University, Zagazig, Egypt. ${ }^{4}$ Biochemistry Department, Faculty of Science, Zagazig University, Zagazig, Egypt.

\section{Received: 12 January 2021 Accepted: 4 April 2021}

Published online: 14 May 2021

\section{References}

1. Wei L, Xiao Y, Li L, Xiong X, Han Y, Zhu X, Sun L (2018) The susceptibility genes in diabetic nephropathy. Kidney Dis 4(4):226-237. https://doi.org/1 $0.1159 / 000492633$

2. Bhatti A, Usman M (2015) Drug targets for oxidative podocyte injury in diabetic nephropathy. Cureus 7:e393

3. Mehrabzadeh M, Pasalar P, Karimi M, Abdollahi M, Daneshpour M, Asadolahpour E et al (2016) Association between ELMO1 gene polymorphisms and diabetic nephropathy in an Iranian population. J Diabetes Metab Disord 15:43. https://doi.org/10.1186/s40200-016-0265-3 eCollection 2015

4. Marshall SM (2014) Natural history and clinical characteristics of CKD in type 1 and type 2 diabetes mellitus. Adv Chronic Kidney Dis 21:267-272 Back to cited text no. 4

5. Keating ST, El-Osta A (2015) Epigenetics and metabolism. Circ Res 116(4): 715-736. https://doi.org/10.1161/CIRCRESAHA.116.303936

6. Pan L, Lian W, Zhang X, Han S, Cao C, Li X, Li M (2018) Human circular RNA0054633 regulates high glucose-induced vascular endothelial cell dysfunction through the microRNA-218/roundabout 1 and microRNA-218/ heme oxygenase-1 axes. Int J Mol Med 42:597-606. https://doi.org/10.3892/ ijmm.2018.3625

7. Zhao Z, Li X, Jian D, Hao P, Rao L, Li M (2017) Hsa_circ_0054633 in peripheral blood can be used as a diagnostic biomarker of pre-diabetes and type 2 diabetes mellitus. Acta Diabetol 54(3):237-245. https://doi.org/10.1 007/s00592-016-0943-0

8. Kato M (2018) Noncoding RNAs as therapeutic targets in early-stage diabetic kidney disease. Kidney Res Clin Pract 37(3):197-209. https://doi. org/10.23876/j.krcp.2018.37.3.197

9. Tabaei BP, Al-Kassab AS, llag LL, Zawacki CM, Herman WH (2001) Does microalbuminuria predict diabetic nephropathy? Diabetes Care 24(9):15601566. https://doi.org/10.2337/diacare.24.9.1560

10. American Diabetes Association (2020) 6. Glycemic targets: standards of medical care in diabetes-2020. Diabetes Care 43:S111-S134. https://doi. org/10.2337/dc20-S010

11. Silveiro SP, Araújo GN, Ferreira MN, Souza FD, Yamaguchi HM, Camargo EG (2011) Chronic Kidney Disease Epidemiology Collaboration (CKD-EPI) equation pronouncedly underestimates glomerular filtration rate in type 2 diabetes. Diabetes Care 34(11):23-28

12. Friedewald WT, Levy RI, Fredrickson DS (1972) Estimation of the concentration of low-density lipoprotein cholesterol in plasma, without use of the preparative ultracentrifuge. Clin Chem 18(6):499-502. https://doi. org/10.1093/clinchem/18.6.499
13. Gerstein HC, Mann JF, Yi Q, Zinman B, Dinneen SF, Hoogwerf B, Halle JP, Young J, Rashkow A, Joyce C, Nawaz S, Yusuf S (2001) Albuminuria and risk of cardiovascular events, death, and heart failure in diabetic and nondiabetic individuals. JAMA 286(4):421-426. https://doi.org/10.1001/ja ma.286.4.421

14. Livak KJ, Schmittgen TD (2001) Analysis of relative gene expression data using real-time quantitative PCR and the $2-\Delta \Delta C T$ method. Methods 25(4): 402-408

15. Xu J, Zou MH (2009) Molecular insights and therapeutic targets for diabetic endothelial dysfunction. Circulation 120(13):1266-1286. https://doi.org/10.11 61/CIRCULATIONAHA.108.835223

16. Kern TS (2007) Contributions of inflammatory processes to the development of the early stages of diabetic retinopathy. Exp Diabetes Res 2007:1-14. https://doi.org/10.1155/2007/95103

17. Zelmanovitz T, Gerchman F, Balthazar APS, Thomazelli FCS, Matos JD, Canani LH (2009) Diabetic nephropathy. Diabetol Metab Syndr 1(1):10. https://doi.org/10.1186/1758-5996-1-10

18. Murussi MA, Coester JL, Gross SPS (2003) Diabetic nephropathy in type 2 diabetes mellitus. Arq Bras Endocrinol Metabol 47(3):207-219. https://doi. org/10.1590/S0004-27302003000300003

19. Elnajjar MM, Dawood AE, Salem MA, Kasemy ZA, Nohman OT (2016) Diabetic nephropathy among diabetic patients attending El Mahalla General Hospital. J Egypt Soc Nephrol Transplant 16:39-43

20. Pavkov ME, Knowler WC, Bennett PH, Looker HC, Krakoff J, Nelson RG (2006) Increasing incidence of proteinuria and declining incidence of end-stage renal disease in diabetic Pima Indians. Kidney Int 70(10):1840-1846. https:// doi.org/10.1038/sj.ki.5001882

21. Kathryn AK, McClellan WM, Ziemer DC, Kleinbacim DG, Bori JR (2000) Risk factors for microalbuminuria in black Americans with newly diagnosed type 2 diabetes. Am J Kidney Dis 36:903-913

22. ADVANCE Collaborative Group, Patel A, MacMahon S (2008) Intensive blood glucose control and vascular outcomes in patients with type 2 diabetes. $\mathrm{N}$ Engl J Med 358:2560

23. Duckworth W, Abraira C, Moritz T, Reda D, Emanuele N, Reaven PD, Zieve FJ, Marks J, Davis SN, Hayward R, Warren SR, Goldman S, McCarren M, Vitek ME, Henderson WG, Huang GD (2009) Glucose control and vascular complications in veterans with type 2 diabetes. N Engl J Med 360(2):129139. https://doi.org/10.1056/NEJMoa0808431

24. Klein R, Klein BEK, Moss SE (1997) Is obesity related to microvascular and macrovascular complications in diabetes - the Wisconsin Epidemiologic Study of Diabetic Retinopathy. Arch Intern Med 157(6):650-656. https://doi. org/10.1001/archinte.1997.00440270094008

25. Haupt E, Benecke A, Haupt A, Herrmann R, Vogel H, Walter C (1999) The KID Study Vl: diabetic complications and associated diseases in younger type 2 diabetics still performing a profession. Prevalence and correlation with duration of diabetic state, BMI and C-peptide. Exp Clin Endocrinol Diabetes 107:435-441

26. Fang Y, Wang X, Li W, Han J, Jin J et al (2018) Screening of circular RNAs and validation of circANKRD36 associated with inflammation in patients with type 2 diabetes mellitus. Int J Mol Med. https://doi.org/10.3892/ijmm.2018.3783

27. Zhang Y, Zhang XO, Chen T, Xiang JF, Yin QF, Xing YH, Zhu S, Yang L, Chen LL (2013) Circular intronic long noncoding RNAs. Mol Cell 51:792-806

28. Li F, Zhang L, Li W, Deng J, Zheng J, An M, Lu J, Zhou Y (2015) Circular RNA ITCH has inhibitory effect on ESCC by suppressing the $\mathrm{Wnt} / \beta$-catenin pathway. Oncotarget 6:6001-6013

29. Chamorro-Jorganes A, Araldi E, Suarez Y (2013) MicroRNAs as pharmacological targets in endothelial cell function and dysfunction. Pharmacol Res 75:15-27

30. Fan X, Weng X, Zhao Y, Chen W, Gan T, Xu D (2017) Circular RNAs in cardiovascular disease: an overview. Biomed Res Int 2017:513578

31. Li Z, Huang C, Bao C, Chen L, Lin M, Wang X, Zhong G, Yu B, Hu W, Dai L et al (2015) Exon-intron circular RNAs regulate transcription in the nucleus. Nat Struct Mol Biol 22:256-264

32. Hu W, Han Q, Zhao L (2019) Wang L Circular RNA circRNA_15698 aggravates the extracellular matrix of diabetic nephropathy mesangial cells via miR-185/TGF- $\beta 1$. J Cell Physiol 234(2):1469-1476

\section{Publisher's Note}

Springer Nature remains neutral with regard to jurisdictional claims in published maps and institutional affiliations. 